\title{
Adsorption of HCN at the Surface of Ice. A Grand Canonical Monte Carlo Simulation Study
}

\author{
Milán Szőri ${ }^{1}$ and Pál Jedlovszky ${ }^{2,3,4}$
}

${ }^{1}$ Department of Chemical Informatics, Faculty of Education, University of Szeged, Boldogasszony sgt. 6. H-6725 Szeged, Hungary

${ }^{2}$ Laboratory of Interfaces and Nanosize Systems, Institute of Chemistry, Eötvös Loránd University, Pázmány P. Stny 1/A, H-1117 Budapest, Hungary

${ }^{3}$ MTA-BME Research Group of Technical Analytical Chemistry, Szt. Gellért tér 4, H-1111 Budapest, Hungary

${ }^{4}$ EKF Department of Chemistry, Leányka utca 6, H-3300 Eger, Hungary

Running title: HCN Adsorption on Ice

e-mail: milan@jgypk.u-szeged.hu (M.Sz.), pali@ chem.elte.hu (P.J.) 


\begin{abstract}
The adsorption of HCN molecules at the surface of hexagonal $\left(\mathrm{I}_{\mathrm{h}}\right)$ ice is studied under tropospheric conditions by a set of grand canonical Monte Carlo simulations. Although the adsorption isotherm is of Langmuir shape and the saturated adsorption layer is practically monomolecular, lateral interactions are found to have a non-negligible effect on the adsorption. The shape of the isotherm can be rationalized by the fact that the interaction energy for $\mathrm{HCN}$-water and $\mathrm{HCN}-\mathrm{HCN}$ pairs is so close to each other and therefore monomolecular adsorption with lateral interaction, turns into a special case. At low surface coverages the $\mathrm{HCN}$ molecules prefer a tilted orientation, pointing by the $\mathrm{N}$ atom flatly toward the ice surface, in which they can form a strong $\mathrm{O}-\mathrm{H}^{\cdots \cdots} \mathrm{N}$ type hydrogen bond with the surface water molecules. At high surface coverages, an opposite tilted orientation is preferred, in which the $\mathrm{H}$ atom points toward the ice phase and the $\mathrm{HCN}$ molecule can form only a weak $\mathrm{C}-\mathrm{H}^{\cdots \cdots} \mathrm{O}$ type hydrogen bond with a surface water molecule. This orientational change is dictated by the smaller surface area occupied by a $\mathrm{H}$ than a $\mathrm{N}$ atom, and the corresponding energy loss is (over) compensated by the formation of $\mathrm{C}-\mathrm{H}^{\cdots \cdots} \mathrm{N}$ type hydrogen bonds between neighboring HCN molecules. The obtained results have several consequences both on the atmospheric effect of $\mathrm{HCN}$ and also on the possible prebiotic formation of precursor molecules of adenine. These consequences are also discussed in the paper.
\end{abstract}




\section{Introduction}

Hydrogen cyanide $(\mathrm{HCN})$ is a highly water soluble but weak acid $\left(\mathrm{p} K_{\mathrm{a}}=9.2\right)$ and its effect on life is manifold. First of all, tissue disruption of cyanogenic glycosides and cyanolipids synthesizing plant species (including many economically important food plants) causes liberating the respiratory poison hydrogen cyanide. This phenomenon of cyanogenesis accounts for numerous cases of acute and chronic cyanide poisoning of animals and man. [!1] Despite its known toxicity, large quantity of $\mathrm{HCN}$ (ca. $0.40 \mathrm{Tg}(\mathrm{N}) / \mathrm{yr}$ ) is manufactured for applications as fumigants and chemical intermediates, [!2] and its direct anthropogenic release is stringently controlled. However, biomass burning has been identified as the main source of atmospheric organic nitrogen in the form of HCN. The emission estimate of HCN from biomass burning flux is in the range of 0.6-3.2 $\mathrm{Tg}(\mathrm{N}) / \mathrm{yr}$. [!3] Hydrogen cyanide degrades very slowly in the atmosphere via reaction with photochemically generated hydroxyl radicals and it is expected to be resistant to direct photolysis. Its estimated half-life is 334 days. Since $\mathrm{HCN}$ is very soluble, its wet deposition is the most probable removal mechanism and then biologically consumed. [!4,5] While $\mathrm{HCN}$ is a tracer of biomass burning pollution, its atmospheric and biogeochemical cycles are poorly understood. [!2] Therefore, there is a need to better quantify its global budget. In wet deposition, HCN can be captured by droplets or cloud ice crystals through collision. The effectiveness of the collision is controlled by the adsorption of $\mathrm{HCN}$ on either aqueous or icy surface, despite their importance, to our best knowledge, adsorption properties has not been characterized yet.

Besides its environmental importance, $\mathrm{HCN}$ is also considered as a key component in abiotic formation of simple organic compounds containing nitrogen which are essential building blocks of living cells. [!6] For instance, synthesis of adenine by the condensed or gas phase oligomerization of $\mathrm{HCN}$ is of particular interest because adenine is essential nucleobase and formally the pentamer of HCN. [!7-9] The spread presence of HCN and its tautomer form hydrogen isocyanide (HNC) in interstellar clouds [!10] also may suggest the possibility of the gas-phase synthesis of adenine in those clouds. Besides nucleobases, amino acids or peptides can also be synthesized through the oligomerization of HCN followed by hydrolysis, [!11] but its thermodynamics interpretation needs further investigation. [!12] Several mechanisms have been proposed for the oligomerization of $\mathrm{HCN}$ and the structures of stable intermediate oligomers have been sought. In particular, HCN dimers have been the focus of previous experimental and theoretical studies. [!13-15] It is believed that the dimerization represents 
the initial step in the formation of primitive nucleobases or amino acids. Although, theoretical and kinetic results predict that the thermal formation of covalently bounded HCN dimer, iminoacetonitrile $(\mathrm{HN}=\mathrm{CHCN})$, cannot occur efficiently under interstellar conditions even though proton catalysis substantially lowers reaction barriers. [!16] Another issue is that adenine was detected as a reaction product in aqueous solutions only when concentrated HCN (1-11 M) was used. [!17,18] However, in aqueous solutions in which the cyanide concentration is $0.01 \mathrm{M}$ or less $\mathrm{HCN}$ does not form oligomers, instead it hydrolyzes in a stepwise fashion to yield formamide and formic acid. [!7,19] Based on these evidences, it is less plausible that prebiotic formation of the biomolecular building blocks via the oligomerization could happen in the bulk or gas phase. Alternatively, a possible enrichment of the $\mathrm{HCN}$ can occur at interfaces such as air/ice interface. Indeed, Bada intimates the idea of an ice world-based origin of biomolecular building blocks. [!20] The experiments performed demonstrated that the synthesis of aromatic hydrocarbons, purines and pyrimidines and other nitrogen heterocycles of potential prebiotic interest is favored in the ice matrix by classical HCN pathways following a freezing-concentration model. [!21] However, role of the local HCN enrichment is unknown at molecular level. Furthermore, structure of the interfacial $\mathrm{HCN}$ clusters can be served as a springboard for their polymerization and the preferred orientations of $\mathrm{HCN}$ are also important properties since they can be highly affected the polymerization mechanism.

The goal of the present paper is thus to examine the interaction of $\mathrm{HCN}$ with the ice surface at $200 \mathrm{~K}$ (i.e., a temperature which is relevant for both the upper troposphere and probably also for prebiotic evolution of the biomolecular building blocks), on the basis of grand canonical ensemble Monte Carlo (GCMC) simulations. [!22,23] The main advantage of using the GCMC method is that it not only allows the analysis of the energy and surface orientation of the adsorbed molecules at various surface coverages, but it is capable of determining the adsorption isotherm from extremely low pressures up to and above the point of condensation. The GCMC method has already successfully been applied for studying the adsorption of water and other small molecules at a number of different solid surfaces, such as on covalent organic frameworks, [!24-26] carbonaceous materials, [!27-35] ice, [!36-42] metal oxides, [!43-46] kaolinite, [!47-49] zeolites, [!50-57] self-assembled monolayers, [!58,59] and in protein crystals. [!60] Having the adsorption isotherm determined the structure of the adsorption layer, binding energy of the adsorbed molecules as well as the preferred orientations of the adsorbed HCN molecules both relative to the ice surface and to the neighboring $\mathrm{HCN}$ molecules are analyzed in detail at various surface coverages. 
The paper is organized as follows. Details of the simulations performed are given in sec. 2. The results are presented and discussed in detail in terms of the adsorption isotherm, density profile of the adsorption layer, as well as orientation and energetics of the adsorbed molecules in section 3. Finally, in section 4 the main conclusions of this study are summarized.

\section{Computer Simulations}

To model the adsorption of $\mathrm{HCN}$ on $\mathrm{I}_{\mathrm{h}}$ ice a set of 26 Monte Carlo simulations have been performed on the grand canonical $(\mu, V, T)$ ensemble at the tropospheric temperature of $200 \mathrm{~K}$. The chemical potential of $\mathrm{HCN}$ has been systematically varied from simulation to simulation, ranging from -51.81 to $-33.52 \mathrm{~kJ} / \mathrm{mol}$. This chemical potential range covers the entire adsorption process from a few isolated $\mathrm{HCN}$ molecules being bound at the surface to condensation of HCN. Adsorption isotherm is simply obtained as the average number of $\mathrm{HCN}$ molecules in the basic box as a function of their chemical potential. The $X, Y$ and $Z$ axes of the rectangular basic simulation box have been 100.0, 35.926 and $38.891 \AA$ long, respectively, $X$ being the surface normal axis. The basic box has contained 2880 water molecules arranged in 18 molecular layers in the middle of the basic box along its surface normal axis $X$ according to the geometry of the hexagonal $\left(\mathrm{I}_{\mathrm{h}}\right)$ ice crystal in such a way that the free surfaces of the ice phase correspond to the (0001) surface of $I_{h}$ ice.

Water molecules have been modeled by the five-site TIP5P potential. [!61] The choice of this water model was dictated by the fact that it reproduces very well, within 1-3 K the melting point of $\mathrm{I}_{\mathrm{h}}$ ice. [!62,63] HCN molecules have been described by the potential model proposed by Kotdawala et al., [!56] based on the OPLS force field. [!64,65] All molecular models used are rigid and pairwise additive, i.e., the total potential energy of the system is regarded to be simply the sum of the pair interaction energies of the molecules. The interaction energy of a molecule pair is described as the sum of the Lennard-Jones and charge-charge Coulomb interaction energies of the respective sites. The interaction and geometry parameters of the potential models used are summarized in Tables 1 and 2, respectively. All interactions have been truncated to zero at the center-center based cut-off distance of $12.5 \AA$. In accordance with the original parametrization of the potential models used $[! 61,64,65]$ no long-range correction of the electrostatic interaction has been employed.

The simulations have been done by the program MMC. [!66] In the simulations particle displacement and $\mathrm{HCN}$ insertion/deletion attempts have been made in an alternating 
order. In a particle displacement step a randomly chosen molecule, either water or HCN, has been randomly displaced by no more than $0.25 \AA$ and randomly rotated around a randomly chosen space-fixed axis by no more than $15^{\circ}$. HCN insertion and deletion attempts have been made with $50-50 \%$ probabilities. In these steps either a new HCN molecule was tried to be inserted into the system or a randomly chosen HCN molecule was attempted to be removed from the system. Insertion steps have been made according to the cavity biased insertion scheme of Mezei, [!67,68] i.e., particles have only been attempted to be inserted into empty cavities of the radius of at least $2.5 \AA$. Suitable cavities have been searched for along a $100 \times 100 \times 100$ grid. Particle displacement steps have been accepted or rejected according to the standard Metropolis criterion, [!23,69] whereas insertion/deletion attempts have been accepted according to the acceptance criteria of cavity biasing. [!67,68] In all the simulations at least $10 \%$ of the particle displacement and $0.5 \%$ of the $\mathrm{HCN}$ insertion/deletion attempts have been successful.

All simulations started from the perfect $\mathrm{I}_{\mathrm{h}}$ ice arrangement of the water molecules with two HCN molecules randomly placed into the vapor phase. The system has been equilibrated by performing $10^{8}$ Monte Carlo moves in every simulation. Then, in the production stage the number of the $\mathrm{HCN}$ molecules has been averaged over $2 \times 10^{8}$ equilibrium configurations. Finally, at selected chemical potential values 2500 sample configurations, separated by $2 \times 10^{5}$ Monte Carlo steps each, have been dumped for further analyses.

\section{Results and Discussion}

3.1. Adsorption Isotherm. The obtained adsorption isotherm, i.e., the mean number of $\mathrm{HCN}$ molecules in the basic simulation box, $\left\langle N_{\mathrm{HCN}}\right\rangle$, as a function of the $\mathrm{HCN}$ chemical potential, $\mu_{\mathrm{HCN}}$, is shown in Figure 1; the corresponding data are also collected in Table 3. The isotherm shows a continuous rise up to about $-35.02 \mathrm{~kJ} / \mathrm{mol}$, where the number of $\mathrm{HCN}$ molecules sharply increases by almost an order of magnitude, indicating condensation of $\mathrm{HCN}$. The simulation performed at $\mu_{\mathrm{HCN}}=-34.85 \mathrm{~kJ} / \mathrm{mol}$ has resulted in an intermediate average number of $\mathrm{HCN}$ molecules, the fluctuation of which being 10-20 times larger than that at any other $\mu_{\mathrm{HCN}}$ value, indicating that at this state point both condensed and gas-like phases of $\mathrm{HCN}$ are present in the sampled configurations. Since this is a clear sign of the immediate vicinity of the point of condensation in a constant volume simulation, here we regard this chemical potential value as the point of condensation of $\mathrm{HCN}$. 
Below the point of condensation, i.e., where a real adsorption layer of the HCN molecules is formed at the surface of ice (the total number of $\mathrm{HCN}$ molecules in the basic box have always been practically identical with those being attached to the ice surface) the isotherm shows no plateau-like region of nearly constant $\left\langle N_{\mathrm{HCN}}\right\rangle$ values. This behavior of HCN is similar to that of formaldehyde [!37] and aromatic hydrocarbons, [!42] but it is in a clear contrast with that of several adsorbate molecules having either hydrogen bonding ability or large dipole moment, such as methanol, [!36] formic acid, [!38] acetone [!39] or benzaldehyde. [!40] The lack of such a plateau indicates that no specifically strong adsorption layer of the HCN molecules can be formed at the ice surface, it also suggests that the adsorption layer is always monomolecular. This point is addressed further in the following subsection.

To present the adsorption isotherm also in a more convential form we converted the $\left\langle N_{\mathrm{HCN}}>\right.$ vs. $\mu_{\mathrm{HCN}}$ data to the $\Gamma$ vs. $p_{\text {rel }}$ isotherm, where $\Gamma$ is the surface density of the HCN molecules:

$$
\Gamma=\frac{<N_{\mathrm{HCN}}>}{2 Y Z},
$$

and $p_{\text {rel }}=p / p^{0}$ is the relative pressure, $p$ and $p^{0}$ being the pressure of the system and that of the saturated vapor, respectively. $p_{\text {rel }}$ has been calculated simply as [!45]

$$
p_{\text {rel }}==\frac{\exp \left(\mu_{\mathrm{HCN}} / k_{\mathrm{B}} T\right)}{\exp \left(\mu^{0} / k_{\mathrm{B}} T\right)}
$$

where $k_{\mathrm{B}}$ is the Boltzmann constant and $\mu^{0}=-34.85 \mathrm{~kJ} / \mathrm{mol}$ is the chemical potential value corresponding to the point of condensation. Since the physical meaning of $p_{\text {rel }}$ implies the vapor phase of $\mathrm{HCN}$, the isotherm is only converted to the $\Gamma\left(p_{\text {rel }}\right)$ form below the point of condensation. The $\Gamma$ and $p_{\text {rel }}$ values of the isotherm are also collected in Table 3, whereas the isotherm in its $\Gamma\left(p_{\text {rel }}\right)$ form is shown in the inset of Fig. 1.

The obtained $\Gamma\left(p_{\text {rel }}\right)$ isotherm can be very well fitted to the functional form of the Langmuir isotherm, i.e., [!70,71]

$$
\Gamma=\Gamma_{\max } \frac{p_{\text {rel }} K}{p_{\text {rel }} K+1},
$$


where the two parameters $\Gamma_{\max }$ and $K$ are the surface density of the saturated adsorption layer and the Langmuir partition coefficient, respectively. Fitting eq. 3 to the obtained data resulted in the values of $\Gamma_{\max }=12.7 \pm 0.4 \mu \mathrm{mol} / \mathrm{m}^{2}$ and $K=2.7 \pm 0.2$. The Langmuir curve fitted to the simulated data is also shown in the inset of Fig.1. The Langmuir character of the adsorption isotherm stresses again the monomolecular character of the adsorption layer, and suggests that lateral interactions, i.e., those between two neighboring $\mathrm{HCN}$ molecules might not play a major role in determining the adsorption. This point is further investigated in the following sub-sections.

It should also be noted that the $\Gamma\left(p_{\text {rel }}\right)$ isotherm is an experimentally easily accessible quantity, and hence it could, in principle, serve as a test of the validity of the present computer simulations. Unfortunately, however, we are not aware of any experimental study of the HCN adsorption on ice, and hence this validation of the simulation results cannot be done here.

Based on the behavior of the adsorption isotherm we have chosen four points at which 2500 sample configurations have been dumped and analyzed in detail Thus, systems I and II at $\mu_{\mathrm{HCN}}=-44.33 \mathrm{~kJ} / \mathrm{mol} \quad\left(p_{\text {rel }}=3.35 \times 10^{-3}\right)$ and $\mu_{\mathrm{HCN}}=-39.34 \mathrm{~kJ} / \mathrm{mol} \quad\left(p_{\text {rel }}=6.72 \times 10^{-2}\right)$, respectively, correspond to unsaturated adsorption layers, whereas systems III and IV at $\mu_{\mathrm{HCN}}=-35.02 \mathrm{~kJ} / \mathrm{mol}\left(p_{\mathrm{rel}}=0.91\right)$ and $\mu_{\mathrm{HCN}}=-34.69 \mathrm{~kJ} / \mathrm{mol}$ are just below and above the point of condensation, respectively. An equilibrium snapshot of these systems, both in top and side views are shown in Figure 2 as taken out from the simulations.

3.2. Density Profiles. The number density profile of HCN molecules along the surface normal axis $X$ is shown in Figure 3 as obtained in systems I-IV. For reference, the density profile of the water molecules in system $I$ is also shown in the inset of the figure. For calculating these profiles the position of the $\mathrm{HCN}$ and water molecules have been approximated by that of their $\mathrm{C}$ and $\mathrm{O}$ atoms, respectively. The profiles are averaged over the two ice surfaces present in the basic simulation box.

As is seen, the adsorption layer is indeed monomolecular in systems I and II, and almost monomolecular even in system III, i.e., right below the point of condensation, where only a very small second peak of the profile is seen. Integration of this profile up to the minimum between the two peaks at $|X|=35.7 \AA$ reveals that only about $8 \%$ of the $\mathrm{HCN}$ molecules give rise to the second peak, and hence belong to the second molecular layer above the ice surface. These findings confirm that the adsorption layer is indeed practically monomolecular in almost the entire existence range of the vapor phase of $\mathrm{HCN}$, whereas the 
appearance of a small trace of the second molecular layer before the point of condensation also stresses, in accordance with the shape of the $\left\langle N_{\mathrm{HCN}}\right\rangle\left(\mu_{\mathrm{HCN}}\right)$ adsorption isotherm (Fig. 1) the fact the adsorption layer does not show a particular stability even at the stage of a saturated monolayer.

The HCN density profile obtained in system IV, i.e., after the condensation of HCN shows pronounced oscillations across the entire basic box, exhibiting at least six marked layers of the $\mathrm{HCN}$ molecules above the ice surface. The broadness and overlap of these density peaks are clear signs of the disordered character of the condensed HCN phase, whereas the long-ranged layering structure reflects the low temperature of $200 \mathrm{~K}$ of the system. The fact that the position of both the first minimum and the second maximum of this profile coincides with those of the profile of system III clearly confirm that the second, small density peak in system III indeed reflects the appearance of traces of a second molecular layer of HCN above the ice surface. In the following analyses we always limit ourselves to the HCN molecules belonging to the first molecular layer, being in direct contact with the ice phase, i.e., located in the $X$ range of $|X|<35.7 \AA$.

\subsection{Orientation of the Adsorbed Molecules.}

3.3.1. Orientation Relative to the Ice Surface. To characterize the preferred orientation of the adsorbed HCN molecules relative to the ice surface we have calculated the cosine distribution of the angle $\vartheta$, formed by the vector pointing along the $\mathrm{HCN}$ molecule from its $\mathrm{H}$ to $\mathrm{N}$ atom ( $\underline{H N}$ vector) and the surface normal vector $\underline{X}$ pointing away from the ice phase. The distribution of $\cos \vartheta$ is shown in Figure 4 as obtained in systems I-IV. The definition of the angle $\vartheta$ is also illustrated in this figure.

As is seen, in the unsaturated systems the $P(\cos \vartheta)$ distribution has a peak around the $\cos \vartheta$ value of -0.2 , corresponding to $\vartheta \approx 100^{\circ}$. Thus, the adsorbed HCN molecules prefer a nearly parallel alignment with the ice surface, tilted by only $10^{\circ}$ from it, pointing by the $\mathrm{N}$ atom flatly toward the ice phase. Considering also the fact that in two of their four preferred orientations at the ice surface the water molecules point flatly, by about $15^{\circ}$ away from the ice phase by one or both of their O-H bonds (see orientations A and D in Fig. 9 of Ref. [!36]), the observed preference of $\mathrm{HCN}$ orientation can be well explained by the possibility of forming a hydrogen bond between the O-H group of a surface water molecule of either of these two orientations and the $\mathrm{N}$ atom of the $\mathrm{HCN}$ molecule. Furthermore, the sticking out $\mathrm{H}$ of the orientation B is also easily accessible for the nitrogen of HCN. Such hydrogen bonded water- 
HCN pairs (I-III) are shown in Figure 5.a as taken out from equilibrium snapshots of the simulations.

The orientational preferences of the adsorbed HCN molecules change slightly upon saturation of the first molecular layer. Thus, in system III, although any alignment corresponding to a $\cos \vartheta$ value between -0.5 and 0.5 (i.e., to a tilt angle from the surface plane smaller than $30^{\circ}$ ) occurs with relatively high probability, the most probable orientation now corresponds to $\cos \vartheta \approx 0.2$, i.e., $\vartheta \approx 80^{\circ}$. In this orientation the $\mathrm{H}$ atom of the HCN molecule points flatly toward the ice surface, and hence the adsorbed HCN molecule can form a weak, C-H donated hydrogen bond with a surface water molecule pointing by one or both of its lone pair directions flatly away from bulk ice (see orientations A, C and D in Fig. 9 of Ref. [!36]). These type of hydrogen bonded water-HCN pairs (IV-VI) are also depicted in Fig. 5.

This change of the orientational preference of the adsorbed HCN molecules upon saturation can be explained by the interplay of energetic and steric effects. Thus, at low coverages when most of the HCN molecules can be adsorbed almost independently from each other the main driving force of the adsorption is to have as low potential energy of the adsorbed molecule as possible. Clearly, an $\mathrm{O}-\mathrm{H}$ donated $\mathrm{O}-\mathrm{H}^{\cdots \cdots} \mathrm{N}$ type hydrogen bond is considerably stronger than a $\mathrm{C}-\mathrm{H}^{\cdots \cdots} \mathrm{O}$ type one, i.e., when the binding $\mathrm{H}$ atom belongs to a $\mathrm{CH}$ rather than an $\mathrm{OH}$ group, which explains the strong preference for the $\cos \vartheta \approx-0.2$ in the unsaturated layer. On the other hand, the small $\mathrm{H}$ atom of the adsorbed $\mathrm{HCN}$ molecule occupies a considerable smaller surface area than its $\mathrm{N}$ atom, hence, as the adsorption layer gets increasingly more crowded the importance of the opposite, i.e., $\cos \vartheta \approx 0.2$ alignment also increases, as witnessed by the gradual decrease of the former and increase of the latter peak upon going from system I to III. Finally, in the first molecular layer of the condensed system IV no clear orientational preference of the $\mathrm{HCN}$ molecules is seen.

3.3.2. Relative Orientation of the Neighboring HCN Molecules. To characterize also the preferred relative alignments of the neighboring adsorbed HCN molecules we have calculated the cosine distribution of the angle $\gamma$, formed by the $\underline{H N}$ vectors of two neighboring HCN molecules. To define when two adsorbed HCN molecules of the first molecular layer can be regarded as neighbors we have calculated the two-dimensional radial distribution function of the $\mathrm{HCN}$ molecules within the first molecular layer (i.e., the normalized lateral density of the $\mathrm{HCN}$ molecules around each other as a function of the distance of their $\mathrm{C}$ atoms within the $Y Z$ plane of the ice surface). The resulting $g_{2 \mathrm{D}}(r)$ functions are shown in Figure 6 
for systems I-IV. As is seen, the sharp first peak of the $g_{2 \mathrm{D}}(r)$ function is followed by a clear minimum at $6 \AA$ in every case. Therefore, here we regard two HCN molecules of the first molecular layer as neighbors if the distance of their $\mathrm{C}$ atoms in the $Y Z$ plane is less than $6 \AA$. It is also important to mention that the first high peak at $3.75 \AA$ indicates a preference of the adsorbed HCN molecules being close vicinity even at low coverages.

The $P(\cos \gamma)$ distribution of the neighboring HCN molecules along with the illustration of the definition of the angle $\gamma$ is shown in Figure 7 as obtained in systems I-IV. As is seen, the distributions obtained in systems II-IV all show a peak at $\cos \gamma=1$, indicating the clear preference of the neighboring $\mathrm{HCN}$ molecules for parallel (head-to-tail) alignment in these systems. This orientational preference is clearly dictated by the dipole-dipole interaction. A HCN pair of such relative orientation is also shown in Fig. 5. However, in the case of system I relative orientations of the neighbors corresponding to a $\cos \gamma$ value above 0.2 seem to be equally probable. Although the corresponding $P(\cos \gamma)$ curve is rather noisy (due to the few neighboring $\mathrm{HCN}$ pairs present at such low surface density) the difference of this distribution from those in systems II-IV is apparent.

To further investigate this point we have recalculated the $P(\cos \gamma)$ distribution in system I with a stricter definition of neighboring molecules, i.e., using the C-C cut-off distance of $3.75 \AA$ A. (This value corresponds, on average, to one nearest neighbor molecule in the saturated layer.) The obtained distribution is shown in the inset of Fig. 7. As is seen, now the distribution has a clear peak between 0.25 and 0.55 , i.e., in the angular range of about $55^{\circ}<\gamma<75^{\circ}$, whereas the preference of the molecules for the parallel alignment completely disappeared. In other words, although first shell lateral HCN neighbors generally prefer the parallel relative alignment, the closest $\mathrm{HCN}$ pairs prefer tilted relative orientations, since they can be hydrogen bonded to each other and both can bind to water molecules in the same time. The alignment of such a near neighbor HCN pair is also shown in Fig. 5.

3.4. Energetic Background of the Adsorption. To characterize the energetic background of the adsorption we have calculated the distribution of the binding energy, $U_{\mathrm{b}}$, of the $\mathrm{HCN}$ molecules at the ice surface. $U_{\mathrm{b}}$ is simply the sum of the total potential energy an adsorbed $\mathrm{HCN}$ molecule experiences at the ice surface, in other words, the energy cost of bringing this molecule to infinite distance. Since the potential energy of the system has been pairwise additive in the simulations, $U_{\mathrm{b}}$ can simply be calculated as the sum of the pair interaction energies between the given $\mathrm{HCN}$ molecule and all the other molecules in the 
system. Besides the total binding energy, $U_{\mathrm{b}}$, the distribution of its contributions coming from the ice phase, $U_{\mathrm{b}}^{\text {wat }}$, and from the other $\mathrm{HCN}$ molecules, $U_{\mathrm{b}}^{\mathrm{HCN}}$, (i.e., the sum of the pair interaction energies between the given $\mathrm{HCN}$ molecule and the water molecules, and all the other HCN molecules, respectively) have also been calculated.

The $P\left(U_{\mathrm{b}}\right), P\left(U_{\mathrm{b}}^{\text {wat }}\right)$ and $P\left(U_{\mathrm{b}}^{\mathrm{HCN}}\right)$ distributions are shown in Figure 8 as obtained in the first molecular layer of HCN in systems I-IV. The $P\left(U_{\mathrm{b}}^{\text {wat }}\right)$ distribution exhibits a sharp and clear peak at $-39 \mathrm{~kJ} / \mathrm{mol}$ in system I. The position of this peak indicates that at low surface coverage $\mathrm{HCN}$ molecules can rather strongly be bound to the ice surface. Considering the fact that the pair interaction energy of a hydrogen bonded TIP5P water pair is below $-20 \mathrm{~kJ} / \mathrm{mol}$, [!61] we can conclude that this strong binding is based on one single but rather strong $\mathrm{O}-\mathrm{H}^{\cdots \cdots} \mathrm{N}$ type hydrogen bond the $\mathrm{HCN}$ molecule can form with the ice phase. This finding is also in a clear accordance with the observed orientational preference of the HCN molecules in this system (see Fig. 4). The position of this peak at $-39 \mathrm{~kJ} / \mathrm{mol}$ can also serve as an estimate of the heat of adsorption at infinite dilution, a quantity that could also be measured experimentally. However, as it was already mentioned above, we are not aware of any experimental investigation of this system, and hence no such comparison of our value with any experimental data can be done at the moment.

It is also seen that with increasing surface concentration the peak of $P\left(U_{\mathrm{b}}^{\text {wat }}\right)$ gradually shifts to higher (i.e., less negative) energies. Thus, the position of the peak is at $-32 \mathrm{~kJ} / \mathrm{mol}$ in system II and around $-22 \mathrm{~kJ} / \mathrm{mol}$ in systems III and IV, i.e., when the ice surface is already saturated. This shift reflects the increasing competition of the adsorbed HCN molecules at the surface, evidenced also in the increasing preference of a tilted orientation in which the $\mathrm{H}$ rather than the $\mathrm{N}$ atom of the $\mathrm{HCN}$ molecule points toward the ice surface (see Fig. 4). This change in the orientational preference of the $\mathrm{HCN}$ molecules upon saturation indicates the increasing dominance of the weak, $\mathrm{C}-\mathrm{H}^{\cdots \cdots} \mathrm{O}$ type hydrogen bonds between the adsorbed molecules and the ice surface. The increasing preference of these weak C-H ${ }^{\cdots \cdots} \mathrm{O}$ type hydrogen bonds instead of the strong $\mathrm{O}-\mathrm{H}^{\cdots \cdots} \mathrm{N}$ type ones is reflected in the gradual shift of the $P\left(U_{\mathrm{b}}^{\text {wat }}\right)$ peak to higher energies upon saturation.

The $P\left(U_{\mathrm{b}}^{\mathrm{HCN}}\right)$ distribution exhibits a bimodal structure in the unsaturated systems I and II. A trivial peak of these distributions occurs at zero energy, reflecting the presence of $\mathrm{HCN}$ molecules that are isolated from the other ones in the adsorption layer. The other peak 
around $-13 \mathrm{~kJ} / \mathrm{mol}$ is given by $\mathrm{HCN}$ molecules having a near $\mathrm{HCN}$ neighbor. Surprisingly, this peak is rather high even in system I; integration up to the minimum between the two peaks at $-5 \mathrm{~kJ} / \mathrm{mol}$ reveals that about $15 \%$ of the $\mathrm{HCN}$ molecules give rise to this peak and only the other $85 \%$ are isolated from the other adsorbed molecules. This finding indicates that even at very low surface density the adsorbed HCN molecules have a tendency of selfassociation. The rise, broadening and extension down to about $-40 \mathrm{~kJ} / \mathrm{mol}$ of this peak in (the still unsaturated) system II clearly indicates that, in spite of the observed Langmuir shape of the isotherm (see the inset of Fig. 1) the adsorption of the individual HCN molecules is not independent of each other, instead, $\mathrm{HCN}$ molecules have a preference for being adsorbed in the vicinity of each other. The energetic driving force of this preference, as evidenced by the peak position of the $P\left(U_{\mathrm{b}}^{\mathrm{HCN}}\right)$ distribution and also by the preferred parallel (head-to-tail) alignment of the neighboring HCN molecules (see Fig. 7) is the possibility of the formation of a $\mathrm{C}-\mathrm{H}^{\cdots \cdots} \mathrm{N}$ type hydrogen bond between two neighboring $\mathrm{HCN}$ molecules. The fact that is system II the distribution extends well below the double of this peak position also suggests the possibility that some of the HCN molecules can even participate in two of such hydrogen bonds (once as the $\mathrm{H}$-acceptor and once as the $\mathrm{H}$-donor partner).

Upon saturation, the distribution gets broader and shifts to even lower energies, reflecting the appearance of a large number of surrounding $\mathrm{HCN}$ molecules around each other. Finally, condensation leads to a further shift of the $P\left(U_{\mathrm{b}}^{\mathrm{HCN}}\right)$ distribution to lower energies due to the interaction with the large number of non-first layer $\mathrm{HCN}$ molecules in the system.

The evolution of the total binding energy distribution, $P\left(U_{\mathrm{b}}\right)$, with increasing surface density reflects the interplay of the observed two opposite effects, i.e., the weakening of the interaction with the ice phase and the increase of the lateral interactions. Thus, in system I the $P\left(U_{\mathrm{b}}\right)$ distribution has its peak at $-40 \mathrm{~kJ} / \mathrm{mol}$, just slightly below the peak position of the $P\left(U_{\mathrm{b}}^{\text {wat }}\right)$ distribution of $-39 \mathrm{~kJ} / \mathrm{mol}$. Although in system II the $P\left(U_{\mathrm{b}}\right)$ peak appears at about the same energy value as in system I, the distribution clearly broadens to lower energies. Finally, saturation of the surface leads to a clear shift of the entire distribution to lower energies; its peak in system III, i.e., in the case of the saturated adsorption layer occurs at $39.34 \mathrm{~kJ} / \mathrm{mol}$. These results indicate that the energy loss of the adsorbed HCN molecules due to the weaker interaction with the ice phase (e.g., due to the increasing dominance of the $\mathrm{C}-\mathrm{H}^{\cdots \cdots} \mathrm{O}$ instead of $\mathrm{O}-\mathrm{H}^{\cdots \cdots} \mathrm{N}$ type hydrogen bonds) upon saturation is well overcompensated 
by the increasing lateral interaction of the adsorbed molecules (e.g., by the increasing number of $\mathrm{C}-\mathrm{H}^{\cdots \cdots} \mathrm{N}$ type hydrogen bonds between the neighboring $\mathrm{HCN}$ molecules). All these findings clearly point out that, in spite of the Langmuir shape of the adsorption isotherm, lateral interactions play a key role in the adsorption of $\mathrm{HCN}$ molecules at the ice surface.

\section{Summary and Conclusions}

In this paper we have presented a detailed analysis of the adsorption of $\mathrm{HCN}$ at the surface of $I_{h}$ ice under tropospheric conditions on the basis of a set of grand canonical Monte Carlo simulations. Although we have calculate here several quantities, such as the adsorption isotherm itself, the Langmuir partition coefficient, the surface density of the saturated adsorption layer, and the heat of adsorption at infinite dilution, that are easily accessible also by experimental methods, and hence could be used to validate the results of the present simulations, in the lack of existing experimental data such a validation cannot be done right now. Nevertheless, this fact clearly stresses the urgent need of relevant experiments in this field.

We have found that the adsorption isotherm is of Langmuir shape and, correspondingly, the saturated adsorption layer is practically monomolecular. However, in spite of the Langmuir shape of the isotherm, lateral interactions are found to play an important role in the adsorption. Thus, HCN molecules are preferentially adsorbed in the vicinity of each other, even at low surface coverages. Since interaction energy of HCN-HCN (-19.21 $\mathrm{kJ} / \mathrm{mol})$ and $\mathrm{HCN}-\mathrm{H}_{2} \mathrm{O}(-20.34 \mathrm{~kJ} / \mathrm{mol})$ are comparable, [!72] the thermodynamic driving force of this lateral self-association is the energy gain accompanying the formation of weak, $\mathrm{C}-\mathrm{H}^{\cdots \cdots} \mathrm{N}$ type hydrogen bonds between the neighboring HCN molecules. Correspondingly, neighboring $\mathrm{HCN}$ molecules are found to prefer parallel, head-to-tail type relative arrangement. However, a different relative orientational preference is found among the closest neighbors, in particular, at low surface coverage, i.e., a strongly tilted arrangement in which the axes of the two molecules form an angle of $55^{\circ}-75^{\circ}$.

It is also found that the adsorbed HCN molecules prefer slightly tilted orientations relative to the ice surface. At low surface coverages the preferred alignment is such that the $\mathrm{N}$ atom of the molecule points toward the ice surface, while in the saturated adsorption layer an opposite alignment with a slightly outward oriented $\mathrm{N}$ atom is found to be preferred. Considering also the orientational preferences of the surface water molecules [!36] the former $\mathrm{HCN}$ alignment allows the formation of a strong, $\mathrm{O}-\mathrm{H}^{\cdots \cdots} \mathrm{N}$ type, whilst the latter one that of a 
weak, $\mathrm{C}-\mathrm{H}^{\cdots \cdots} \mathrm{O}$ type hydrogen bond with a water molecule at the ice surface. The preference of the latter orientation at high surface coverage can be explained by the smaller surface area occupied by a contact $\mathrm{H}$ than a $\mathrm{N}$ atom, and the energetic loss corresponding to the formation of a weak $\mathrm{C}-\mathrm{H}^{\cdots \cdots} \mathrm{O}$ type hydrogen bond instead of a strong $\mathrm{O}-\mathrm{H}^{\cdots \cdots} \mathrm{N}$ type one is overcompensated by the relatively strong lateral interactions, in particular, the formation of $\mathrm{C}-\mathrm{H}^{\cdots \cdots} \mathrm{N}$ type hydrogen bonds between the large number of adsorbed HCN molecules.

Finally, the implications of the obtained results both on atmospheric processes and on the possible abiotic formation of adenine have to be discussed. The heat of adsorption of HCN at infinitely low surface coverage has turned to be about $-39 \mathrm{~kJ} / \mathrm{mol}$. This value is considerably lower than what was previously found for other adsorbates that can also form one single hydrogen bond with the ice surface, such as formaldehyde $(-27 \mathrm{~kJ} / \mathrm{mol})$ [!37] or acetaldehyde $(-34 \mathrm{~kJ} / \mathrm{mol}),[! 41]$ indicating also the particular strength of the $\mathrm{O}-\mathrm{H}^{\cdots \cdots} \mathrm{N}$ type hydrogen bond formed by an adsorbed HCN and a surface water molecule. Considering also the relative abundance of $\mathrm{HCN}$ at the troposphere and the good solubility of $\mathrm{HCN}$ in water, implying that once the ice grain containing $\mathrm{HCN}$ at its surface gets melt it dissolves these $\mathrm{HCN}$ molecules, the atmospheric importance of this adsorption process cannot be neglected.

From the point of view of the possible formation of iminoacetonitrile, i.e., a precursor molecule of adenine in interstellar conditions, the low surface coverage states are of relevance. It has been found that at low surface coverages the HCN molecules prefer such orientations in which the surface water molecules can provide them a proton through an $\mathrm{O}-\mathrm{H}^{\cdots \cdots} \mathrm{N}$ type hydrogen bond. Proton catalysis is known to substantially lower the energy barrier of the dimerization reaction of $\mathrm{HCN}$ to iminoacetonitrile. Further, in spite of the low surface coverage the $\mathrm{HCN}$ molecules are found to show a tendency of self-association in certain (head-to-tail and tilted) preferred relative orientations. All these findings emphasize the possibility of HCN dimerization at the surface of ice grains at low surface coverages under interstellar conditions. Theoretical verification of this possibility, however, still requires $a b$ initio calculations of the system of specifically arranged $\mathrm{HCN}$ dimers with proton provider water molecules. Relevant arrangements of these molecules for such calculations are readily provided by the present study. Work in this direction is currently in progress.

Acknowledgements. This project is supported by the Hungarian OTKA Foundation under project No. 104234. P.J. is a Szentágothai János fellow of NKPR which is gratefully acknowledged. M.Sz. is Magyary Zoltán fellow supported by the European Union and the 
State of Hungary, co-financed by the European Social Fund in the framework of TÁMOP 4.2.4.A/2-11-1-2012-0001 'National Excellence Program' (A2-MZPD-12-0139). Infrastructural support was provided by the TÁMOP-4.2.2.A-11/1/KONV-2012-0047,"New Functional Materials and Their Biological and Environmental Answers" and TÁMOP4.2.2/C-11/1/KONV-2012-0010 co-financed by the European Union and the European Social Fund. 


\section{References}

(1) Poulton, J. E. Cyanogenesis in Plants. Plant Physiology, 1990, 94, 401-405.

(2) Singh, H. B.; Salas, L.; Herlth, D.; Kolyer, R.; Czech, E.; Viezee, W.; Li, Q.; Jacob, D. J.; Blake, D.; Sachse, G.; Harward, C. N.; Fuelberg, H.; Kiley, C. M.; Zhao, Y.; Kondo, Y. In Situ Measurements of $\mathrm{HCN}$ and $\mathrm{CH}_{3} \mathrm{CN}$ over the Pacific Ocean: Sources, Sinks, and Budgets. J. Geophys. Res. - Atmos. 2003, 108, 8795-8808.

(3) Lobert, J. M.; Scharffe, D. H.; Hao, W. M.; Kuhlbusch, T. A.; Seuwen, R.; Warneck, R.; Crutzen, P. J. Experimental Evaluation of Biomass Burning: Nitrogen and Carbon Containing Compounds. In Global Biomass Burning; Levine, J. S., Ed.; MIT Press: Cambridge, 1991, pp. 289-304.

(4) Möller, D. Chemistry of the Climate System; Walter de Gruyter: Berlin, 2010.

(5) Ghosh, R. S.; Ebbs, S. D.; Bushey, J. T.; Neuhauser E. F.; Wong-Chong, E. F. Cyanide Cycle in Nature. In Cyanides in Water and Soil; Dzombak, D. A.; Ghosh, R. S.; Wong-Chong, G. M., Eds.; CRC Press: Boca Raton, 2006, pp. 226-236.

(6) Holm, N. G.; Neubeck, A. Reduction of Nitrogen Compounds in Oceanic Basement and its Implications for HCN Formation and Abiotic Organic Synthesis. Geochem. Trans. 2009, 10:9-1-11.

(7) Ferris, J. P.; Hagan, W. J. HCN and Chemical Evolution: The Possible Role of Cyano Compounds in Prebiotic Synthesis. Tetrahedron 1984, 40, 1093-1120.

(8) Smith, I.W.M.; Talbi, D.; Herbst, E. The Production of HCN Dimer and More Complex Oligomers in Dense Interstellar Clouds. Astron. Astrophys. 2001, 369, 611615.

(9) Roy, D.; Najafian, K.; von Ragué Schleyer, P. Chemical Evolution: The Mechanism of the Formation of Adenine under Prebiotic Conditions. Proc. Natl. Acad. Sci. USA 2007, 104, 17272-17277.

(10) Amino Acids, Peptides and Proteins in Organic Chemistry. Vol.1 Origins and Synthesis of Amino Acids, Hughes, A. B., Ed.; Wiley-VCH: Weinheim, 2009.

(11) Rehder, D. Chemisty in Space: From Intersteller Matter to the Origin of Life; WileyVCH: Weinheim, 2010.

(12) Szőri, M.; Jójárt, B.; Izsák, R.; Szőri, K.; Csizmadia, I. G.; Viskolcz, B. Chemical Evolution of Biomolecule Building Blocks. Can Thermodynamics Explain the 
Accumulation of Glycine in the Prebiotic Ocean? Phys. Chem. Chem. Phys., 2011, 13, 7449-7458.

(13) Savee, J. D.; Mann, J. E.; Continetti, R. E. Two-Body Dissociative Charge Exchange Dynamics of sym-Triazine. J. Phys. Chem. A 2009, 113, 8834-8838.

(14) Evans, R. A.; Lacombe, S. M.; Simon, M. J.; Pfister-Guillouzo, G.;Wentrup, C. Hydrogen Cyanide Dimers: Photoelectron Spectrum of Iminoacetonitrile. J. Phys. Chem. 1992, 96, 4801-4804.

(15) Jobst, K. J.; Hanifa, M. R.; Ruttink, P. J. A.; Terlouw, J. K. The Covalently Bound $\mathrm{HC} \equiv \mathrm{N}$ Dimer Ions $\mathrm{HC}=\mathrm{N}-\mathrm{N}=\mathrm{CH} \bullet^{+}$and $\mathrm{HC}=\mathrm{N}-\mathrm{C}(=\mathrm{N}) \mathrm{H}^{\bullet}{ }^{+}$Are Stable Species in the Gas-Phase, But the Neutral Counterparts Are Not. Chem. Phys. Letters 2009, 473, 257-262.

(16) Yim, M. K.; Choe J. C. Dimerization of $\mathrm{HCN}$ in the Gas Phase: A Theoretical Mechanistic Study. Chem. Phys. Letters 2012, 538, 24-28.

(17) Oró, J.; Kamat, J. S. Amino-acid Synthesis from Hydrogen Cyanide under Possible Primitive Earth Conditions. Nature, 1961, 190, 442-443.

(18) Oró, J.; Kimball, A. P. Synthesis of Purines under Possible Primitive Earth Conditions: II. Purine Intermediates from Hydrogen Cyanide. Arch. Biochem. Biophys. 1962, 96, 293-313.

(19) Sanchez, R. A.; Ferris, J. P.; Orgel, L. E. Studies in Prebiotic Synthesis: II. Synthesis of Purine Precursors and amino Acids from Aqueous Hydrogen Cyanide. J. Mol. Biol. 1967, 30, 223-253.

(20) Bada, J. L. How Life Began on Earth: a Status Report. Earth Planet. Sci. Letters, 1994, 226, 1-15.

(21) Menor-Salván, C.; Marín-Yaseli, M. R. Prebiotic Chemistry in Eutectic Solutions at the Water-Ice Matrix. Chem. Soc. Rev., 2012, 41, 5404-5415.

(22) Adams, D. J. Grand Canonical Ensemble Monte Carlo for a Lennard-Jones Fluid. Mol. Phys. 1975, 29, 307-311.

(23) Allen, M. P.; Tildesley, D. J. Computer Simulation of Liquids; Clarendon: Oxford, 1987.

(24) Jung, D. H.; Kim, D.; Lee, T. B.; Choi, S. B.; Yoon, J. H.; Kim, J.; Choi, K.; Choi, S. H. Grand Canonical Monte Carlo Simulation Study on the Catenation Effect on Hydrogen Adsorption onto the Interpenetrating Metal-Organic Frameworks. J. Phys. Chem. B 2006, 110, 22987-22990. 
(25) Garberoglio, G. Computer Simulation of the Adsorption of Light Gases in Covalent Organic Frameworks. Langmuir 2007, 23, 12154-12158.

(26) Ramsahye, N. A.; Maurin, G.; Bourelly, S.; Llewellyn, P. L.; Devic, T.; Serre, C.; Loiseau, T.; Ferey, G. Adsorption of $\mathrm{CO}_{2}$ in Metal Organic Frameworks of Different Metal Centres: Grand Canonical Monte Carlo Simulations Compared to Experiments. Adsorption 2007, 13, 461-467.

(27) Muller, E. A.; Rull, L. F.; Vega, L. F.; Gubbins, K. E. Adsorption of Water on Activated Carbons: a Molecular Dynamics Study. J. Phys. Chem. 1996, 100, 11891196.

(28) Samios, S.; Stubos, A. K.; Kanellopoulos, N. K.; Cracknell, R. F.; Papadopoulos, G. K.; Nicholson, D. Determination of Micropore Size Distribution from Grand Canonical Monte Carlo Simulations and Experimental $\mathrm{CO}_{2}$ Isotherm Data. Langmuir 1997, 13, 2795-2802.

(29) Muller, E. A.; Hung, F. R.; Gubbins, K. E. Adsorption of Water Vapor-Methane Mixtures on Activated Carbons. Langmuir 2000, 16, 5418-5424.

(30) Challa, S. R.; Sholl, D. S.; Johnson, J. K. Adsorption and Separation of Hydrogen Isotopes in Carbon Nanotubes: Multicomponent Grand Canonical Monte Carlo Simulations. J. Chem. Phys. 2002, 116, 814-824.

(31) Striolo, A.; Chialvo, A. A.; Gubbins, K. E.; Cummings, P. T. Water in Carbon Nanotubes: Adsorption Isotherms and Thermodynamic Properties from Molecular Simulation. J. Chem. Phys. 2005, 122, 234712-1-14.

(32) Moulin, F.; Picaud, S.; Hoang, P. N. M.; Pártay, L. B.; Jedlovszky, P. A Grand Canonical Monte Carlo Simulation Study of Water Adsorption on a Model Soot Particle. Mol. Simul. 2006, 32, 487-493.

(33) Moulin, F.; Picaud, S.; Hoang, P. N. M.; Jedlovszky, P. Grand Canonical Monte Carlo Simulation of the Adsorption Isotherms of Water Molecules on a Model Soot Particle J. Chem. Phys. 2007, 127, 164719-1-11.

(34) Hantal, Gy.; Picaud, S.; Hoang, P. N. M.; Voloshin, V. P.; Medvedev, N. N.; Jedlovszky, P. Water Adsorption Isotherms on Porous Onionlike Carbonaceous Particles. Simulations with the Grand Canonical Monte Carlo Method. J. Chem. Phys. 2010, 133, 144702-1-12.

(35) Firlej, L. ; Kuchta, B. ; Lazarewicz, A. ; Pfeifer, P. Increased $\mathrm{H}_{2}$ Gravimetric Storage Capacity in Truncated Carbon Slit Pores Modeled by Grand Canonical Monte Carlo. Carbon 2013, 53, 208-215. 
(36) Jedlovszky, P.; Partay, L.; Hoang, P. N. M.; Picaud, S.; von Hessberg, P.; Crowley, J. N. Determination of the Adsorption Isotherm of Methanol on the Surface of Ice. An Experimental and Grand Canonical Monte Carlo Simulation Study. J. Am. Chem. Soc. 2006, 128, 15300-15309.

(37) Hantal, Gy.; Jedlovszky, P.; Hoang, P. N. M.; Picaud, S. Calculation of the Adsorption Isotherm of Formaldehyde on Ice by Grand Canonical Monte Carlo Simulation. $J$. Phys. Chem. C 2007, 111, 14170-14178.

(38) Jedlovszky, P.; Hantal, Gy.; Neuróhr, K.; Picaud, S.; Hoang, P. N. M.; von Hessberg, P.; Crowley, J. N. Adsorption Isotherm of Formic Acid on The Surface of Ice, as Seen from Experiments and Grand Canonical Monte Carlo Simulation. J. Phys. Chem. C 2008, 112, 8976-8987.

(39) Hantal, Gy.; Jedlovszky, P.; Hoang, P. N. M.; Picaud, S. Investigation of the Adsorption Behavior of Acetone at The Surface of Ice. a Grand Canonical Monte Carlo Simulation Study. Phys. Chem. Chem. Phys. 2008, 10, 6369-6380.

(40) Petitjean, M.; Hantal, Gy.; Chauvin, C.; Mirabel, P.; Le Calvé, S.; Hoang, P. N. M.; Picaud, S.; Jedlovszky, P. Adsorption of Benzaldehyde at the Surface of Ice, Studied by Experimental Method And Computer Simulation. Langmuir 2010, 26, 9596-9606.

(41) Darvas, M.; Lasne, J.; Laffon, C.; Parent, P.; Picaud, S.; Jedlovszky, P. Adsorption of Acetaldehyde on Ice as Seen From Computer Simulation and Infrared Spectroscopy Measurements. Langmuir 2012, 28, 4198-4207.

(42) Mészár, Zs. E.; Hantal, Gy.; Picaud, S.; Jedlovszky, P. Adsorption of Aromatic Hydrocarbon Molecules at the Surface of Ice, As Seen by Grand Canonical Monte Carlo Simulation. J. Phys. Chem. C 2013, 117, 6719-6729.

(43) Puibasset, J.; Pellenq, R. J. M. Water Adsorption on Hydrophilic Mesoporous and Place Silica Substrates: a Grand Canonical Monte Carlo Simulation Study. J. Chem. Phys. 2003, 118, 5613-5622.

(44) Puibasset, J.; Pellenq, R. J. M. Water Adsorption in Disordered Mesoporous Silica (Vycor) at $300 \mathrm{~K}$ and $650 \mathrm{~K}$ : A Grand Canonical Monte Carlo Simulation Study of Hysteresis. J. Chem. Phys. 2005, 122, 094704-1-10.

(45) Daub, C. D.; Patey G. N.; Jack, D. B.; Sallabi A. K. Monte Carlo Simulations of the Adsorption of $\mathrm{CO}_{2}$ on the $\mathrm{MgO}(100)$ Surface. J. Chem. Phys. 2006, 124, 114706-1-9.

(46) Tombácz, E.; Hajdú, A.; Illés, E.; László, K.; Garberoglio, G.; Jedlovszky, P. Water in Contact with Magnetite Nanoparticles, as Seen from Experiments and Computer Simulations. Langmuir 2009, 25, 13007-13014. 
(47) Rutkai, G.; Kristóf, T. Molecular Simulation Study of Intercalation of Small Molecules in Kaolinite. Chem. Phys. Letters 2008, 462, 269-274.

(48) Croteau, T.; Bertram, A. K.; Patey, G. N. Adsorption and Structure of Water on Kaolinite Surfaces: Possible Insight into Ice Nucleation from Grand Canonical Monte Carlo Calculations. J. Phys. Chem. A 2008, 112, 10708-10712.

(49) Croteau, T.; Bertram, A. K.; Patey, G. N. Simulation of Water Adsorption on Kaolinite under Atmospheric Conditions. J. Phys. Chem. A 2009, 113, 7826-7833.

(50) Jameson, C. J.; Jameson, K.; Baello, B. I.; Lim, H. M. Grand Canonical Monte Carlo Simulations of the Distribution and Chemical Shifts of Xenon in the Cages of Zeolite NaA. 1. Distribution and Xe-129 Chemical Shifts. J. Chem. Phys. 1994, 100, 59655976.

(51) Jameson, C. J.; Jameson, K.; Lim, H. M.; Baello, B. I. Grand Canonical Monte Carlo Simulations of the Distribution and Chemical Shifts of Xenon in the Cages of Zeolite NaA. 2. Structure of the Adsorbed Fluid. J. Chem. Phys. 1994, 100, 5977-5987.

(52) Smit, B. Grand Canonical Monte Carlo Simulations of Chain Molecules: Adsorption Isotherms of Alkanes in Zeolites. Mol. Phys. 1995, 85, 153-172.

(53) Pellenq, R. J. M.; Tavitian, B.; Espinat, D.; Fuchs, A. H. Grand Canonical Monte Carlo Simulations of Adsorption of Polar and Nonpolar Molecules in NaY Zeolite. Langmuir 1996, 12, 4768-4783.

(54) Macedonia, M. D.; Maginn, E. J. Pure and Binary Component Sorption Equilibria of Light Hydrocarbons in the Zeolite Silicalite from Grand Canonical Monte Carlo Simulations. Fluid Phase Equil. 1999, 158-160, 19-27.

(55) Rutkai, G.; Csányi, É.; Kristóf, T. Prediction of Adsorption Equilibria of WaterMethanol Mixtures in Zeolite NaA by Molecular Simulation. Mol. Simul. 2006, 32 , 869-875.

(56) Kotdawala, R. R.; Ozgur Yazaydin, A.; Kazantzis, N.; Thompson, R. W. A Molecular Simulation Approach to the Study of Adsorption of Hydrogen Cyanide and Methyl Ethyl Ketone in Silicalite, Mordenite and Zeolite Beta Structures. Mol. Simul. 2007, $33,843-850$.

(57) Kristóf, T.; Csányi, É.; Rutkai, G. Prediction of Adsorption and Separation of WaterAlcohol Mixtures with Zeolite NaA. Microporous Mesoporous Mat. 2008, 114, 455464.

(58) Szőri, M.; Jedlovszky, P.; Roeselová, M. Water Adsorption on Hydrophilic and Hydrophobic Self-Assembled Monolayers as Proxies For Atmospheric Surfaces. A 
Grand Canonical Monte Carlo Simulation Study. Phys. Chem. Chem. Phys. 2010, 12, 4604-4616.

(59) Szőri, M.; Roeselová, M.; Jedlovszky, P. Surface Hydrophilicity-Dependent Water Adsoprtion on Mixed Self-Assembled Monolayers of $\mathrm{C}_{7}-\mathrm{CH}_{3}$ and $\mathrm{C}_{7}-\mathrm{COOH}$ residues. A Grand Canonical Monte Carlo Simulation Study. J. Phys. Chem. C 2011, 115, 19165-19177.

(60) Resat, H.; Mezei, M. Grand Canonical Monte Carlo Simulation of Water Positions in Crystal Hydrates. J. Am. Chem. Soc. 1994, 116, 7451-7452.

(61) Mahoney, M.; Jorgensen, W. L. A Five-Site Model for Liquid Water and the Reproduction of The Density Anomaly by Rigid, Nonpolarizable Potential Functions. J. Chem. Phys. 2000, 112, 8910-8922.

(62) Nada, H.; van der Eerden, J. P. M. J. An Intermolecular Potential Model for the Simulation of Ice and Water near the Melting Point: A Six-Site Model of $\mathrm{H}_{2} \mathrm{O} . J$. Chem. Phys. 2003, 118, 7401-7413.

(63) Vega, C.; Sanz, E.; Abascal, J. L. F. The Melting Temperature of the Most Common Models of Water. J. Chem. Phys. 2005, 122, 114507-1-9.

(64) Jorgensen, W. L.; Briggs, J. M. Monte Carlo Simulations of Liquid Acetonitrile with a Three-Site Model. Mol. Phys. 1988, 63, 547-558.

(65) Jorgensen, W. L.; Maxwell, D. S.; Tirado-Rives, J. Development and Testing of the OPLS All-Atom Force Field on Conformational Energetics and Properties of Organic Liquids. J. Am. Chem. Soc. 1996, 118, 11225-11236.

(66) Mezei, M. MMC: Monte Carlo Program for Simulation of Molecular Assemblies. URL: http://inka.mssm.edu/ mezei/mmc.

(67) Mezei, M. A Cavity-Biased (T, V, $\mu$ ) Monte Carlo Method for the ComputerSimulation of Fluids. Mol. Phys. 1980, 40, 901-906.

(68) Mezei, M. Mol. Phys. Grand Canonical Ensemble Monte Carlo Study of Dense Liquid Lennard-Jones, Soft Spheres and Water. 1987, 61, 565-582. Erratum: 1989, 67, 12071208.

(69) Metropolis, N.; Rosenbluth, A. W.; Rosenbluth, M. N.; Teller, A. H.; Teller, E. Equation of State Calculations by Fast Computing Machines J. Chem. Phys. 1953, 21 1087-1093.

(70) Langmuir, I. The Constitution and Fundamental Properties of Solids and Liquids. Part I: Solids. J. Am. Chem. Soc. 1916, 38, 2221-2295. 
(71) Shaw, D. J. Introduction to Colloid and Surface Chemistry, Butterworths: London, 1980.

(72) Li, Q.; Wang, X.; Cheng, J.; Li, W.; Gong, B.; Sun, J.:Theoretical Study on the Interlay of Hydrogen Bonds in the Trimers Involving $\mathrm{HCN}$ and Water, Int. J. Quantum Chem., 2009, 109, 1396-1402. 
Table 1. Interaction Parameters of the Molecular Models Used.

\begin{tabular}{ccccc}
\hline \hline molecule & site & $\sigma / \AA$ & $\left(\varepsilon / \mathrm{k}_{\mathrm{B}}\right) / \mathrm{K}$ & $q / \mathrm{e}$ \\
\hline \multirow{3}{*}{ water } & $\mathrm{O}$ & 3.12 & 80.6 & 0.0 \\
& $\mathrm{H}$ & 0.00 & 0.00 & 0.241 \\
& $\mathrm{~L}^{\mathrm{a}}$ & 0.00 & 0.00 & -0.241 \\
& $\mathrm{H}$ & 0.00 & 0.00 & 0.15 \\
$\mathrm{HCN}$ & $\mathrm{C}$ & 3.63 & 77.0 & 0.28 \\
& $\mathrm{~N}$ & 3.20 & 88.0 & -0.43 \\
\hline \hline
\end{tabular}

${ }^{a}$ Non-atomic interaction site.

Table 2. Geometry Parameters of the Molecular Models Used.

\begin{tabular}{ccccc}
\hline \hline molecule & bond & bond length $(\AA)$ & angle & bond angle $(\mathrm{deg})$ \\
\hline \multirow{2}{*}{ water } & $\mathrm{O}-\mathrm{H}$ & 0.9572 & & \\
& $\mathrm{O}-\mathrm{L}$ & 0.7000 & $\mathrm{H}-\mathrm{O}-\mathrm{H}$ & 104.5 \\
& & & $\mathrm{~L}-\mathrm{O}-\mathrm{H}$ & 109.5 \\
& & & $\mathrm{~L}-\mathrm{O}-\mathrm{L}$ & 110.7 \\
$\mathrm{HCN}$ & $\mathrm{H}-\mathrm{C}$ & 1.0680 & & \\
& $\mathrm{C} \equiv \mathrm{N}$ & 1.1570 & & \\
& & & $\mathrm{H}-\mathrm{C} \equiv \mathrm{N}$ & 180.0 \\
\hline \hline
\end{tabular}


Table 3. Data of the Adsorption Isotherm of HCN on Ice, as Obtained From our Simulations.

\begin{tabular}{|c|c|c|c|}
\hline$\mu_{\mathrm{HCN}} / \mathrm{kJ} \mathrm{mol}^{-1}$ & $\left\langle N_{\mathrm{HCN}}\right\rangle$ & $p_{\text {rel }}$ & $\Gamma / \mu \mathrm{mol} \mathrm{m}{ }^{-2}$ \\
\hline-51.81 & 0.070 & $3.72 \times 10^{-5}$ & 0.004 \\
\hline-50.98 & 0.124 & $6.13 \times 10^{-5}$ & 0.007 \\
\hline-50.15 & 0.230 & $1.01 \times 10^{-4}$ & 0.014 \\
\hline-49.32 & 0.338 & $1.67 \times 10^{-4}$ & 0.020 \\
\hline-48.49 & 0.540 & $2.75 \times 10^{-4}$ & 0.032 \\
\hline-47.66 & 0.886 & $4.53 \times 10^{-4}$ & 0.053 \\
\hline-46.83 & 1.47 & $7.47 \times 10^{-4}$ & 0.088 \\
\hline-45.99 & 2.14 & $1.23 \times 10^{-3}$ & 0.127 \\
\hline-45.16 & 3.07 & $2.03 \times 10^{-3}$ & 0.183 \\
\hline$-44.33^{\mathrm{a}}$ & 4.62 & $3.35 \times 10^{-3}$ & 0.275 \\
\hline-43.50 & 6.19 & $5.52 \times 10^{-3}$ & 0.368 \\
\hline-42.97 & 9.93 & $9.10 \times 10^{-3}$ & 0.591 \\
\hline-41.84 & 13.6 & 0.0150 & 0.809 \\
\hline-41.01 & 19.3 & 0.0247 & 1.146 \\
\hline-40.17 & 26.6 & 0.0408 & 1.583 \\
\hline$-39.34^{b}$ & 36.3 & 0.0672 & 2.160 \\
\hline-38.51 & 47.3 & 0.111 & 2.815 \\
\hline-37.68 & 66.6 & 0.183 & 3.962 \\
\hline-36.85 & 93.2 & 0.301 & 5.546 \\
\hline-36.02 & 118 & 0.497 & 7.060 \\
\hline-35.19 & 148 & 0.819 & 8.780 \\
\hline$-35.02^{c}$ & 154 & 0.905 & 9.191 \\
\hline-34.85 & 579 & & \\
\hline$-34.69^{d}$ & 903 & & \\
\hline-34.36 & 907 & & \\
\hline-33.52 & 928 & & \\
\hline
\end{tabular}

${ }^{\mathrm{a}}$ system I ${ }^{\mathrm{b}}$ system II ${ }^{\mathrm{c}}$ system III ${ }^{\mathrm{d}}$ system IV 


\section{Figure legends}

Figure 1. Adsorption isotherm of $\mathrm{HCN}$ on ice, as obtained from our simulations. The inset shows the same isotherm in the more convential surface density vs. relative pressure form. Error bars are only shown when larger than the symbols themselves. The solid black line connecting the data points is just a guide to the eye. The red dashed line in the inset shows the Langmuir isotherm (eq. 3) fitted to the simulated data. Arrows indicate the systems that have been considered for detailed analyses.

Figure 2. Equilibrium snapshot of the adsorbed $\mathrm{HCN}$ molecules at the ice surface as taken out from the simulations in top view (top row) and side view (bottom row). Water oxygens are shown by red sticks; the $\mathrm{H}, \mathrm{C}$ and $\mathrm{N}$ atoms of the $\mathrm{HCN}$ molecules are shown by white, black and blue spheres, respectively. Water hydrogens are omitted for clarity.

Figure 3. Number density profile of the $\mathrm{HCN}$ molecules along the surface normal axis $\mathrm{X}$ as obtained in systems I (blue down triangles), II (orange up triangles), III (red circles), and IV (black squares). Water number density profile is shown by a dashed black line. The inset shows the number density profile of the water molecules in the entire basic simulation box in system I. All the profiles shown are averaged over the two ice surfaces present in the basic box. The dotted vertical line shows the outer boundary of the first molecular layer of HCN.

Figure 4. Cosine distribution of the angle $\vartheta$, formed by the $\underline{H N}$ vector of an adsorbed $\mathrm{HCN}$ molecule and the surface normal vector $\underline{X}$ pointing away from the ice phase, in systems I (blue down triangles), II (orange up triangles), III (red circles), and IV (black squares). The definition of the angle $\vartheta$ is also illustrated (color coding of the HCN atoms is the same as in Figure 2). 


\section{Figure 5.}

Figure 6. Two dimensional radial distribution function of the $\mathrm{HCN}$ molecules in the first molecular layer at the ice surface (calculated considering the distance of the projections of their $\mathrm{C}$ atoms to the $\mathrm{YZ}$ plane) as obtained in systems I (solid blue line), II (dashed orange line), III (dash-dotted red line), and IV (dash-dot-dotted black line). The dotted vertical line shows the boundary of the first (lateral) neighbor shell.

Figure 7. Cosine distribution of the angle $\gamma$, formed by the $\underline{H N}$ vector of two neighboring adsorbed $\mathrm{HCN}$ molecules (i.e., the $\mathrm{C}$ atoms of which are closer to each other in the $Y Z$ plane than $6 \AA$, see the text) in systems I (solid blue line), II (dashed orange line), III (dash-dotted red line), and IV (dash-dot-dotted black line). The inset shows the same distribution considering only the nearest neighbor $\mathrm{HCN}$ molecules (i.e., for which the $\mathrm{C}-\mathrm{C}$ distance in the $Y Z$ plane is less than $3.75 \AA$ ) in system I. The definition of the angle $\gamma$ is also illustrated (color coding of the $\mathrm{HCN}$ atoms is the same as in Figure 2).

Figure 8. Distribution of the total binding energy of an adsorbed $\mathrm{HCN}$ molecule, $U_{\mathrm{b}}$ (i.e., the energy of its interaction with the rest of the system, bottom panel), and its contributions coming from the interaction with the other $\mathrm{HCN}$ molecules, $U_{\mathrm{b}}^{\mathrm{HCN}}$, (middle panel) and with the ice phase, $U_{\mathrm{b}}^{\text {wat }}$, (top panel), as obtained in systems I (solid blue lines), II (dashed orange lines), III (dash-dotted red lines), and IV (dash-dot-dotted black lines). 
Figure 1.

Szőri and Jedlovszky

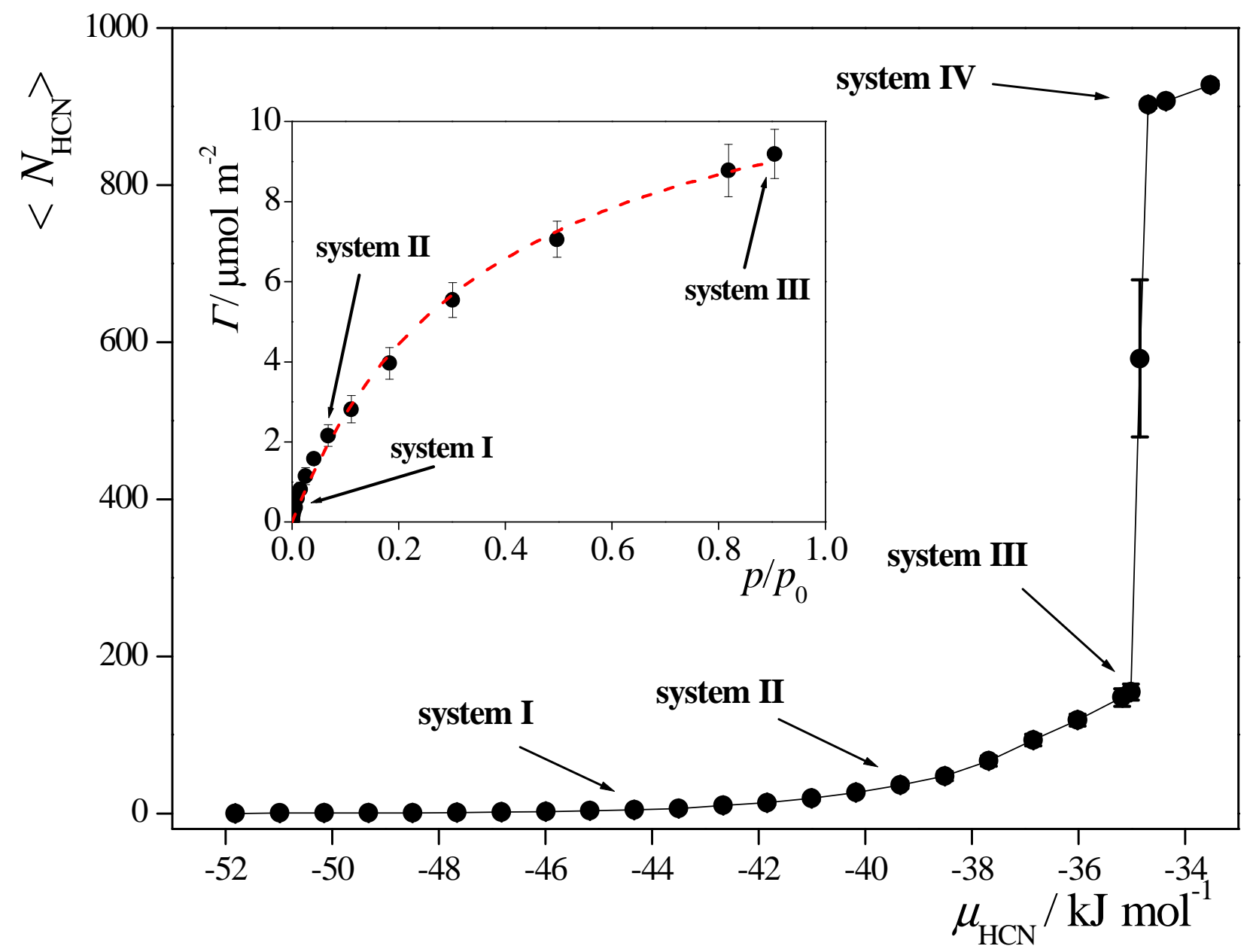


Figure 2.

Szőri and Jedlovszky
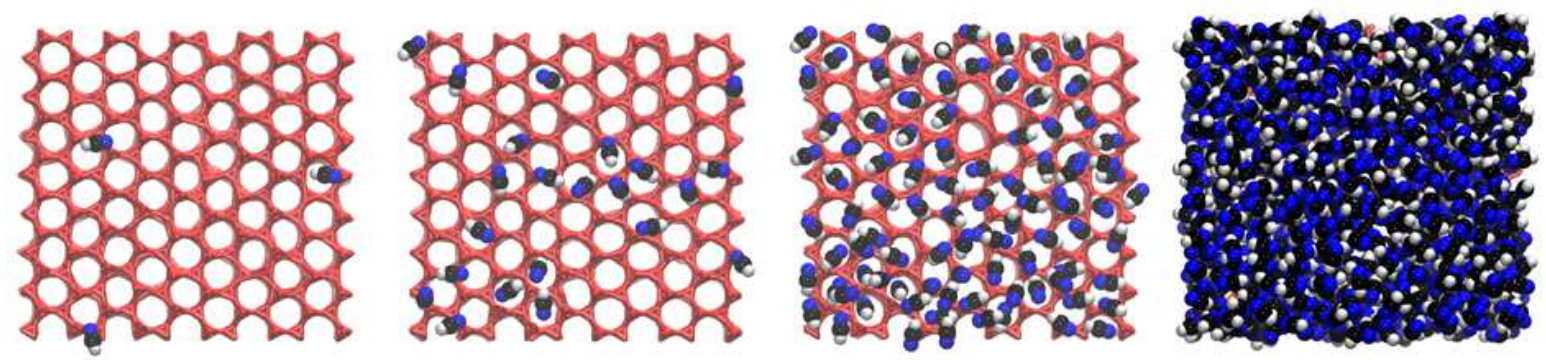

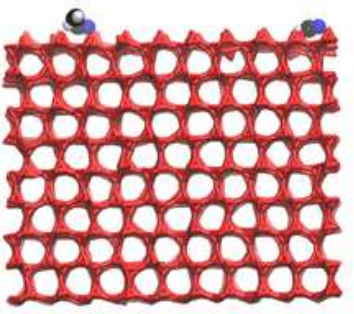

system I

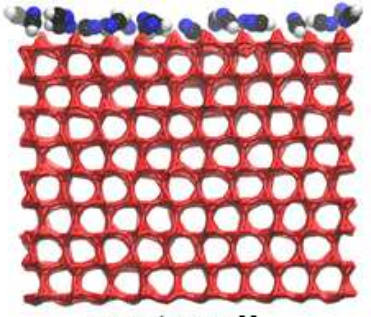

system II

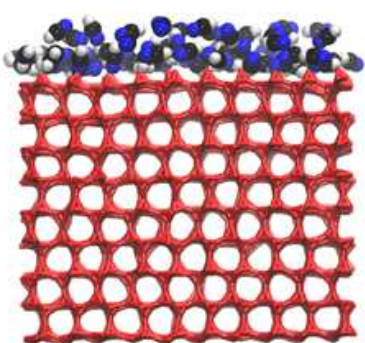

system III

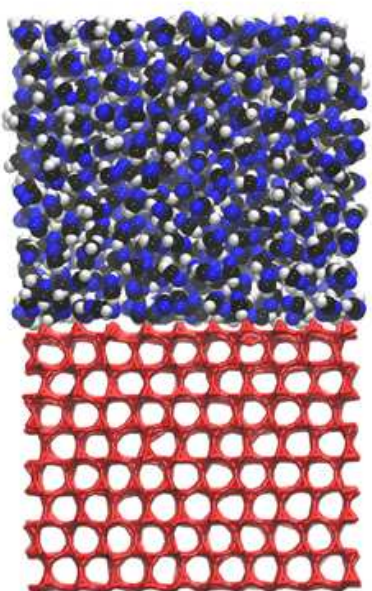

system IV 
Figure 3.

Szőri and Jedlovszky

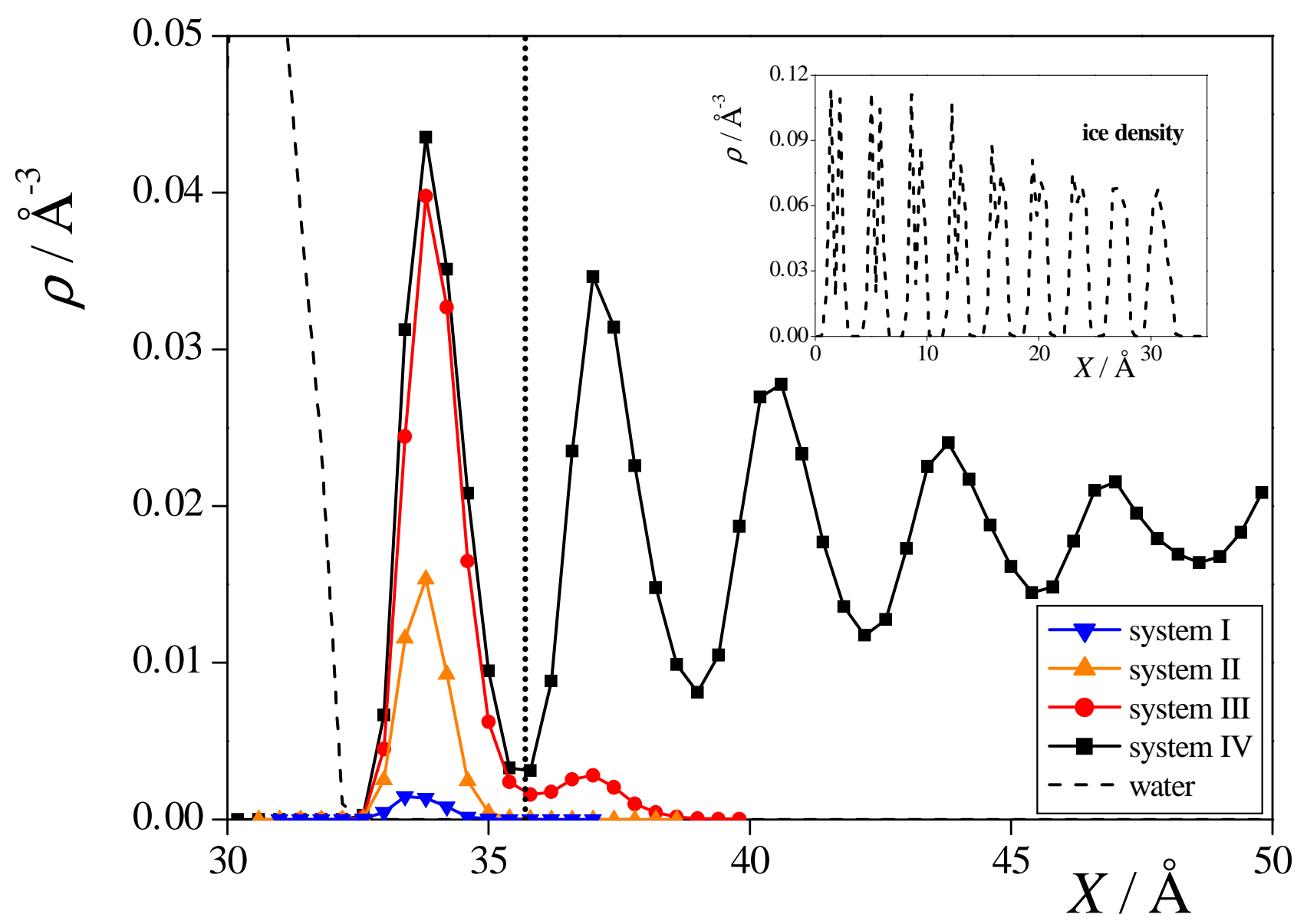


Figure 4.

Szőri and Jedlovszky

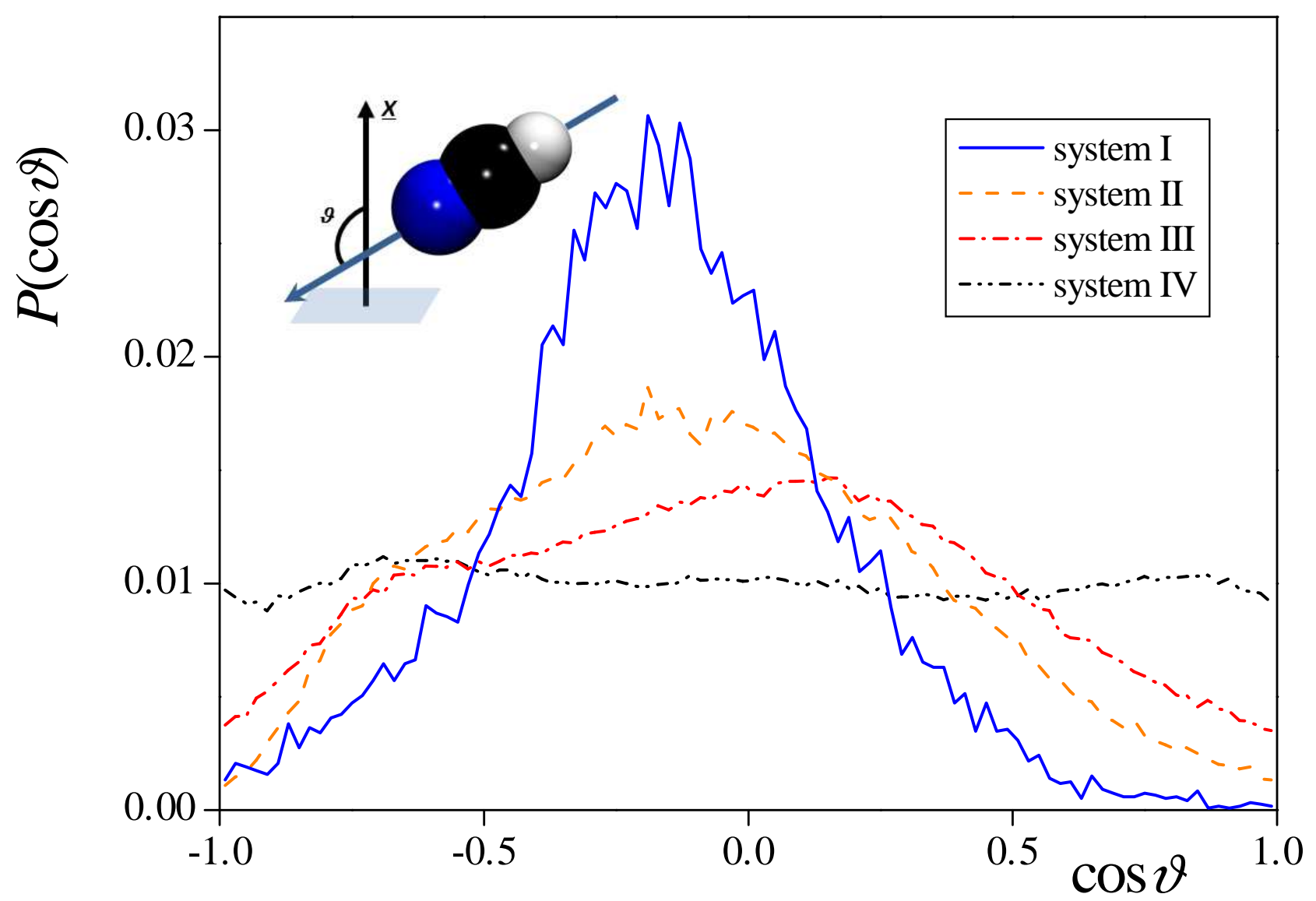


Figure 5.

Szőri and Jedlovszky

(a)
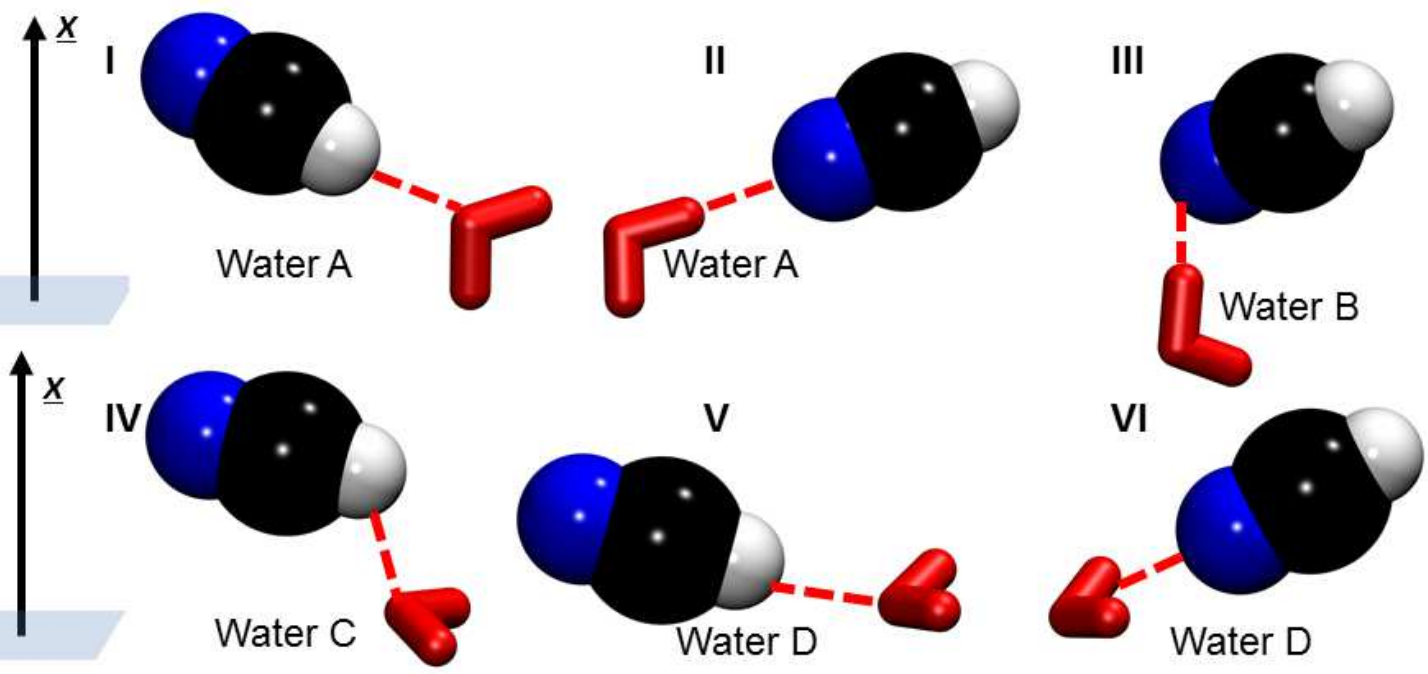

(b)
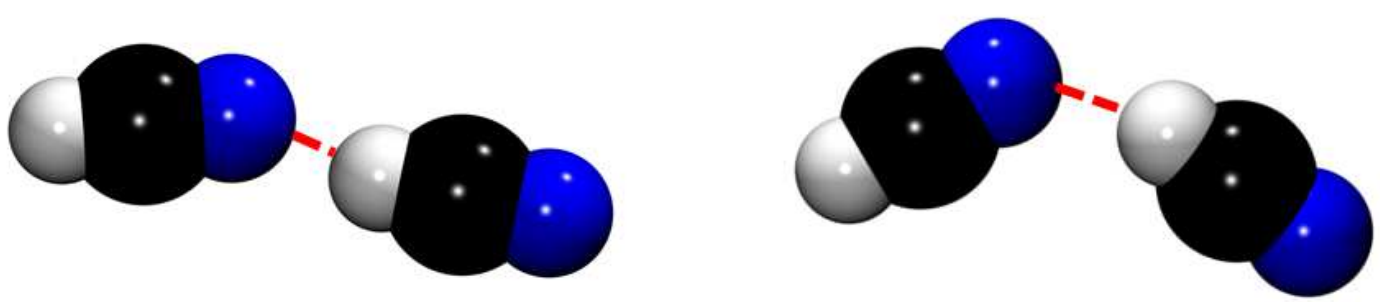
Figure 6.

Szőri and Jedlovszky

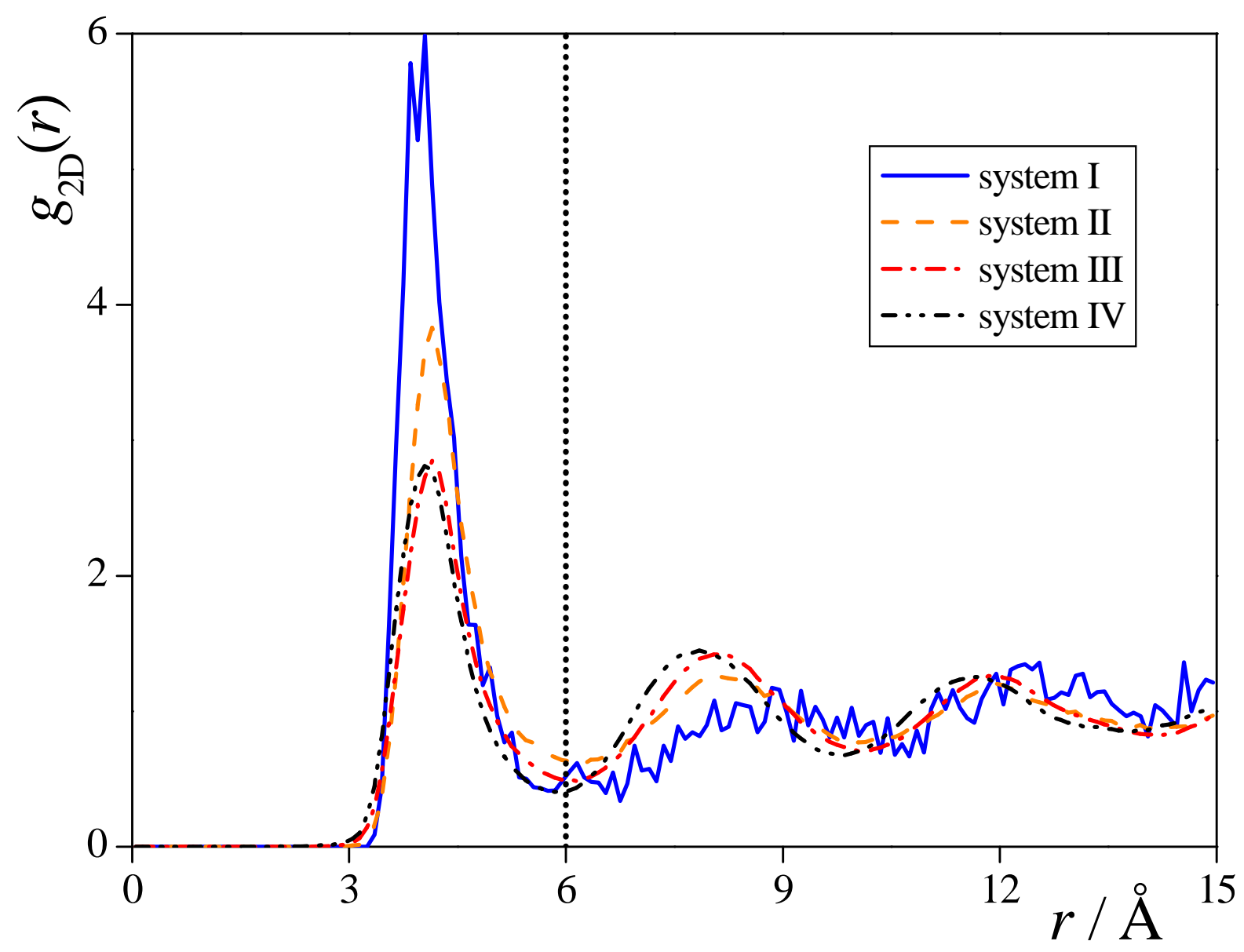


Figure 7.

Szőri and Jedlovszky

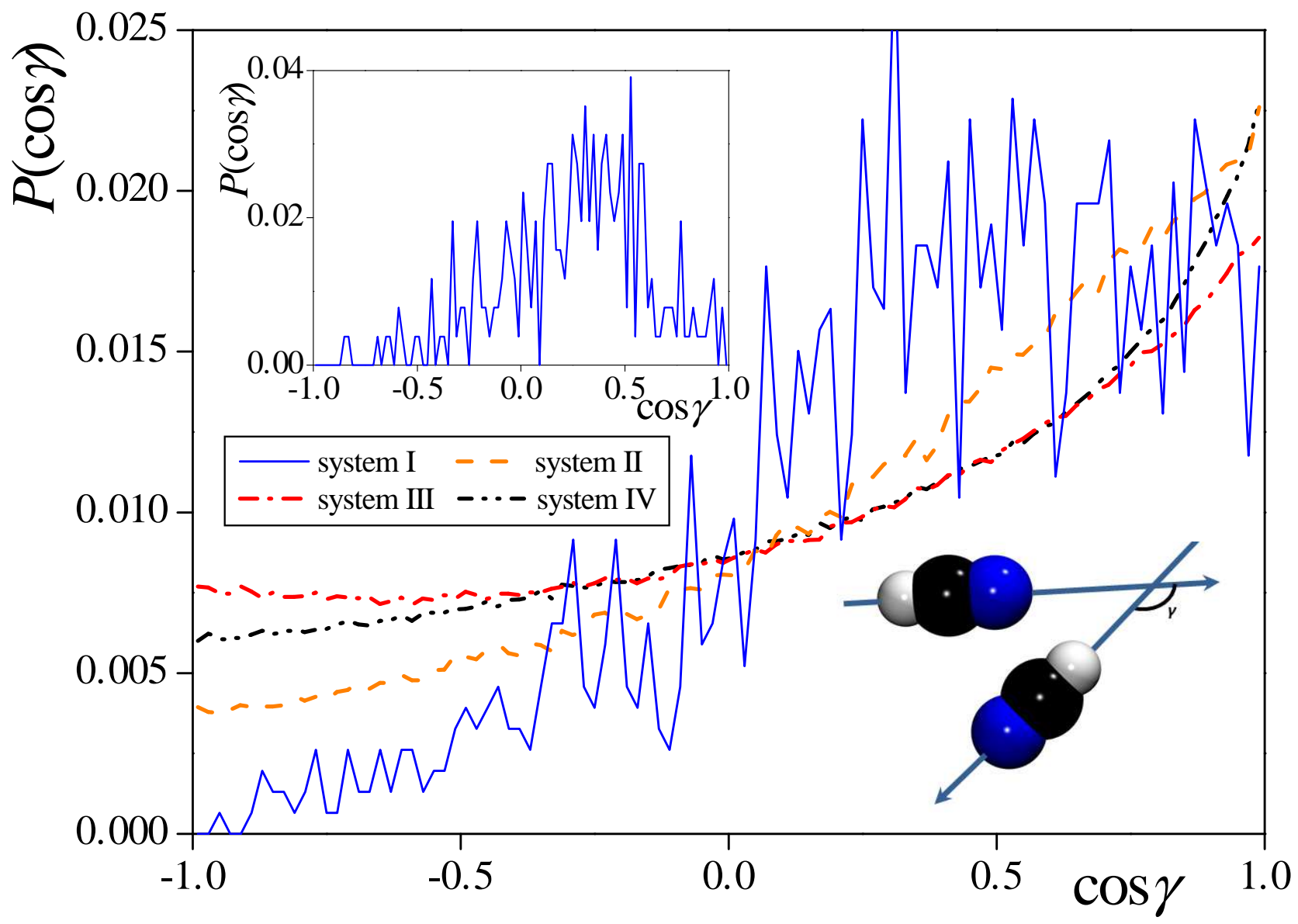


Figure 8.

Szőri and Jedlovszky

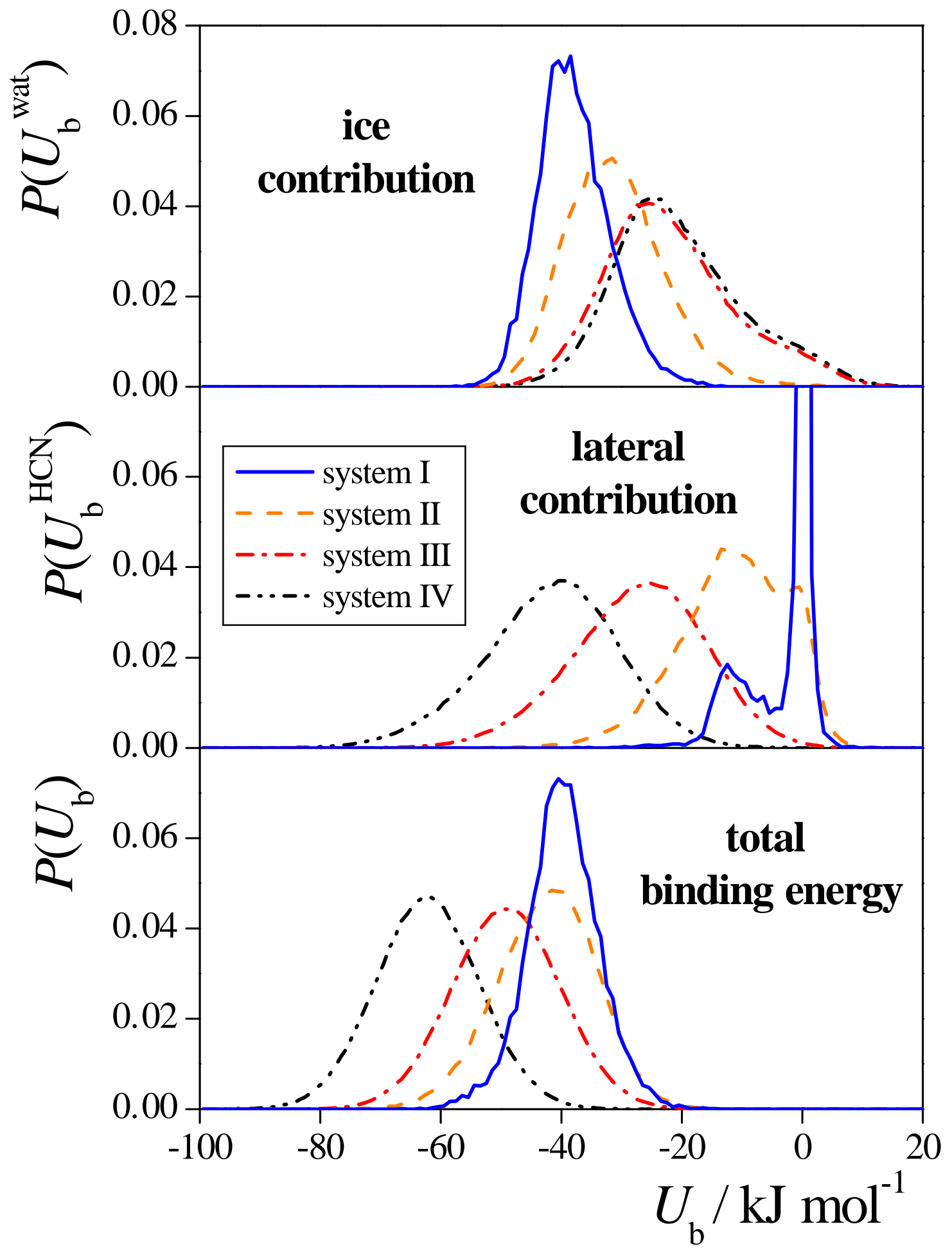


$\underline{\text { Table of Contents Graphics: }}$

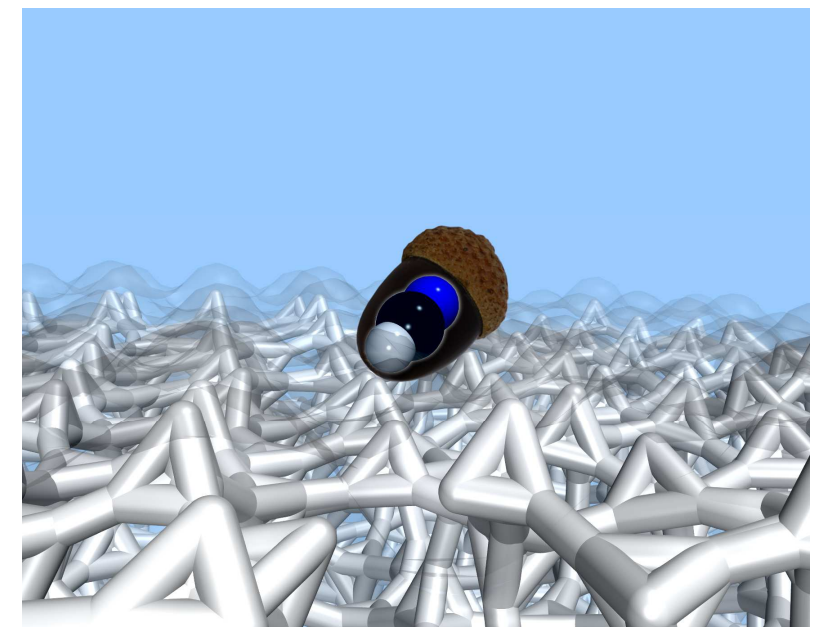

